$$
\left\{\begin{aligned}
\left(\theta_{2}^{2} \theta_{1}\right)^{2} & =P_{5} \\
\theta_{2}^{2} & =P_{7} \\
\theta_{2}^{-1} P_{5} & =P_{6}
\end{aligned}\right\} \text {. }
$$

\title{
REFERENCES
}

1. H. S. M. Coxeter and W. O. J. Moser, Generators and relations for discrete groups, Springer-Verlag, Berlin, 1957.

2. Reuben Sandler, The collineation groups of free planes, Trans. Amer. Math. Soc. 107 (1963), 129-139.

Institute for Defense Analyses, Princeton, New Jersey

\section{PSEUDO-ISOTOPICALLY CONTRACTIBLE SPACES}

O. G. HARROLD, JR.

The space $M$ is called pseudo-isotopically contractible provided that if $A$ is a compact subset of $M$ there is a continuous function $r(x, t)$ of $M \times[0,1]$ into $M$ such that (1) if $t<1, r \mid M \times t$ is a homeomorphism onto, and (2) if $t=1, r \mid A \times 1$ is a point.

Let $X$ be a locally euclidean $n$-dimensional space with the property that each pair of points lies in the interior of some $n$-ball. Clearly $X$ is a connected $n$-manifold without boundary.

Theorem. If $M$ is a locally euclidean $n$-dimensional space with the property that each pair of points lies interior to some $n$-ball, then $M$ is an open $n$-cell if and only if $M$ is pseudo-isotopically contractible.

LEMмa. If $M$ is pseudo-isotopically contractible and $p$ is a point of $M$, the function $r(x, t)$ may be chosen so that $r(A, 1)=p$.

Proof. Let $U$ be the interior of a ball containing $p$ and $q$. Suppose $U$ is given a co-ordinate system $\left(x_{1}, \cdots, x_{n}\right)$, where $x_{1}^{2}+\cdots+x_{n}^{2}<1$ and $\left(x_{1}, \cdots, x_{n}\right) \in \bar{U} \backslash U$ if and only if $x_{1}^{2}+\cdots+x_{n}^{2}=1$.

Let $0<\epsilon<1$; then the mapping

$$
\begin{aligned}
& x_{1}^{\prime}=x_{1}+\epsilon t \rho, \quad 0 \leqq t \leqq 1, \\
& x_{i}^{\prime}=x_{i}, \quad i>1,
\end{aligned}
$$

Received by the editors November 19, 1963. 
where $\rho$ is the distance from $\left(x_{1}, \cdots, x_{n}\right)$ to $\bar{U} \backslash U$, defines an isotopy of $U$ on itself that is fixed on $\bar{U} \backslash U$ and carries the origin into $(\epsilon, 0,0, \cdots, 0)$.

The composition of a finite number of such isotopies will give the desired result.

Proof of the Theorem. Since $M$ is locally separable and connected, it is separable. Since $M$ is also locally compact, $M=\cup_{1}^{\infty} A_{i}$, where $A_{i}$ is compact and $A_{i+1} \supset A_{i}$. Let $B_{i}$ denote the closure of the spherical $1 / i$ neighborhood of $p$.

To each $i=1,2, \cdots$, there is a continuous function $r(x, t ; i)$ on $M \times[0,1]$ that is a homeomorphism onto for $t<1$, and, for $t=1, A_{i}$ is contracted to $p$. Let us choose $t_{i}<1$ such that $r(x, t ; i)$ shrinks $A_{i} \times t_{i}$ to a subset of the interior of $B_{i}$. Suppose $r^{-1}\left(x, t_{i} ; i\right)$ maps $B_{i}$ on $E_{i}$. Then $E_{i}$ is an open $n$-cell and $M=\bigcup_{1}^{\infty} E_{i}$. For, if $q \in M, q \in A_{j}$ for some $j$. Then $r\left(x, t_{j} ; j\right)$ carries $q$ onto $q^{1}$ in $B_{j}$ and $r^{-1}\left(x, t_{j} ; j\right)$ carries $q^{1}$ back onto $q$.

By a recent result of $\mathrm{M}$. Brown, $M$ must be homeomorphic to euclidean $n$-space, $E^{n}[1]$.

\section{REFERENCE}

1. M. Brown, The monotone union of open n-cells is an open n-cell, Proc. Amer. Math. Soc. 12 (1961), 812-814.

UNIVERSITY OF TENNESSEE 